\title{
Tuning the Plasmonic Response up: Hollow Cuboid Metal Nanostructures
}

Aziz Genç, ${ }^{\dagger, \dagger, \S}$ Javier Patarroyo, ${ }^{\dagger, \S}$ Jordi Sancho-Parramon, ${ }^{\S, \|}$ Raul Arenal, ${ }^{\perp, \#}$ Martial Duchamp, $^{\bigcirc}$ Edgar E. Gonzalez, ${ }^{\square}$ Luc Henrard, $\triangle$ Neus G. Bastús, $^{\dagger}$ Rafal E. Dunin-Borkowski, ${ }^{\bigcirc}$ Victor F. Puntes, ${ }^{*} \dagger, 0$ and Jordi Arbiol ${ }^{*}$,

Catalan Institute of Nanoscience and Nanotechnology (ICN2), CSIC and The Barcelona Institute of Science and Technology (BIST), Campus UAB, Bellaterra, 08193 Barcelona, Catalonia, Spain

${ }^{\ddagger}$ Department of Metallurgy and Materials Engineering, Faculty of Engineering, Bartin University, 74100 Bartin, Turkey

"Rudjer Boskovic Institute, Zagreb, Croatia

${ }^{\perp}$ ARAID Fondation, 50018 Zaragoza, Aragon, Spain

\#Laboratorio de Microscopias Avanzadas (LMA), Instituto de Nanociencia de Aragon (INA), Universidad de Zaragoza, 50018

Zaragoza, Spain

OErnst Ruska-Centre for Microscopy and Spectroscopy with Electrons (ER-C) and Peter Grünberg Institute 5 (PGI-5), Forschungszentrum Jülich, D-52425 Jülich, Germany

$\square$ Geophysical Institute, Faculty of Engineering, Pontificia Universidad Javeriana and Nanoscale Science and Technology Centre, 110231, Bogota, Colombia

$\triangle$ Department of Physics, University of Namur, 61, rue de Bruxelles, 5000 Namur, Belgium

-Vall d'Hebron Institut de Recerca (VHIR), 08035 Barcelona, Catalonia, Spain

- Institucio Catalana de Recerca i Estudis Avançats (ICREA), 08010 Barcelona, Catalonia, Spain

Supporting Information

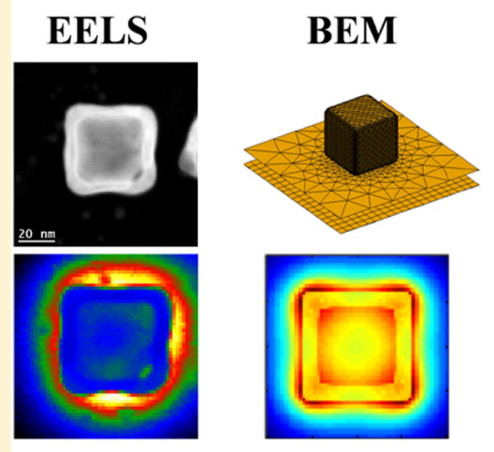

Homogeneous

Plasmon

Resonances

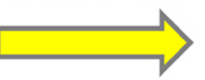

Enhanced

Sensitivity

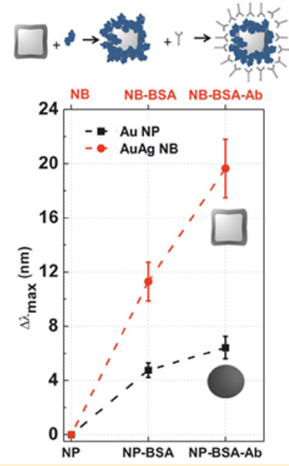

ABSTRACT: We report the fine-tuning of the localized surface plasmon resonances (LSPRs) from ultraviolet to near-infrared by nanoengineering the metal nanoparticle morphologies from solid $\mathrm{Ag}$ nanocubes to hollow $\mathrm{AuAg}$ nanoboxes and $\mathrm{AuAg}$ nanoframes. Spatially resolved mapping of plasmon resonances by electron energy loss spectroscopy (EELS) revealed a homogeneous distribution of highly intense plasmon resonances around the hollow nanostructures and the interaction, that is, hybridization, of inner and outer plasmon fields for the nanoframe. Experimental findings are accurately correlated with the boundary element method (BEM) simulations demonstrating that the homogeneous distribution of the plasmon resonances is the key factor for their improved plasmonic properties. As a proof of concept for these enhanced plasmonic properties, we show the effective label free sensing of bovine serum albumin (BSA) of single-walled AuAg nanoboxes in comparison with solid Au nanoparticles, demonstrating their excellent performance for future biomedical applications.

KEYWORDS: hollow metal nanoparticles, electron energy loss spectroscopy, AuAg, localized surface plasmon resonances, label-free sensing

$\mathrm{M}$ etallic nanostructures have received a great deal of interest due to their ability to generate localized surface plasmon resonances (LSPRs), which are collective oscillations
Received: November 21, 2015

Published: March 16, 2016 

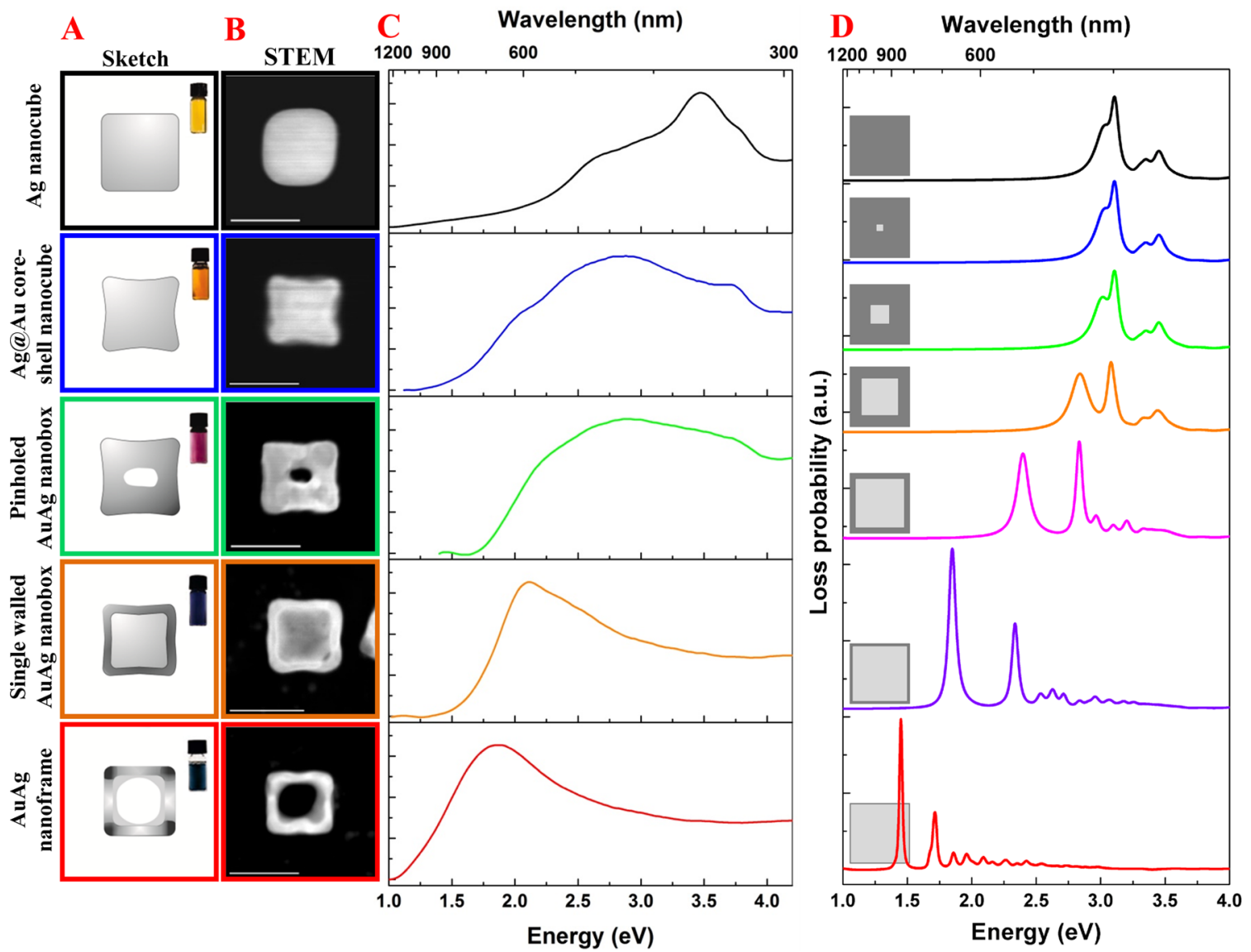

Figure 1. Structural and LSPR evolution of the AuAg nanostructures. (A) Structural sketches and corresponding solution colors of Ag nanocube, Ag@Au core-shell nanocube, pinholed AuAg nanobox, single-walled AuAg nanobox, and AuAg nanoframe (B). HAADF STEM micrographs of the nanostructures where SI EELS have been acquired, from Ag nanocube to AuAg nanoframe (scale bars = $50 \mathrm{~nm}$ ). (C) Zero-loss peak (ZLP) subtracted EEL spectra averaged over the areas of interests, that is, EELS maps, showing the evolution of localized surface plasmon resonances with structural changes. (D) BEM simulated EEL spectra revealing the effect of void size for Ag nanostructures: $50 \mathrm{~nm}$ Ag nanocube (in black), $5 \mathrm{~nm}$ void (in blue), $15 \mathrm{~nm}$ void (in green), $30 \mathrm{~nm}$ void (in orange), $40 \mathrm{~nm}$ void (in magenta), $45 \mathrm{~nm}$ void (in purple), and $48 \mathrm{~nm}$ void (in red).

of conduction electrons of a material excited by an electromagnetic wave. ${ }^{1}$ With the capability of the localization of light at nanoscale, far beyond the diffraction limit of electromagnetic waves in dielectric media, ${ }^{2,3}$ plasmonic nanostructures are attractive building blocks for nano-optics and novel applications such as sensor devices, ${ }^{4}$ surface enhanced Raman spectroscopy (SERS), ${ }^{5}$ photovoltaics, ${ }^{6}$ superlenses, ${ }^{7}$ nanolasers, ${ }^{8}$ invisibility cloaks, ${ }^{9}$ and quantum computing. ${ }^{10}$ It is well-known that the LSPR properties of the metallic nanostructures are affected by their size, shape, composition, and environment, ${ }^{11,12}$ and many different nanostructures have been investigated so far. ${ }^{13}$

$\mathrm{Ag}$ nanostructures are known to have better plasmonic performance than those of $\mathrm{Au}^{14,15}$ however, they are not biocompatible and can easily degrade in most of the biorelated applications. One possible solution in order to avoid Ag is using some other material with higher corrosion resistance (e.g., Au). On the other hand, plasmonic properties of Au nanostructures suffer a lot from the interband transitions as their onset partially overlaps with the LSPRs, causing a decrease in the intensity. ${ }^{16}$ Hollow nanostructures come into prominence in order to overcome this issue as cavities are known to have better plasmonic properties than their solid counterparts thanks to the plasmon hybridization mechanism. ${ }^{17}$ The hybridization of the plasmons results in the enhanced plasmon fields along with more homogeneous distribution as well as the red-shift of plasmon resonances and reduction of LSPR quenching due to absorption. ${ }^{14,15,18,19}$ At this point, nanoengineering plays a crucial role in order to strengthen the plasmonic response of $\mathrm{Au}$ nanocrystals, where hollow morphologies provide biocompatible nanostructures with high plasmonic properties. In order to design these nanostructures with the desired plasmonic features and being able to manipulate LSPRs at the nanoscale, it is essential to understand and locate the plasmon resonances at the nanoscale with the highest possible spatial accuracy. The commonly used techniques such as UV-vis-NIR spectroscopy, dark field microscopy, and near-field scanning optical microscopy (NSOM) exhibit lack of spatial resolution for a complete characterization of local optical features in individual nanostructures. At this point, EELS in a scanning transmission electron microscope (STEM) equipped with a monochromator becomes an ideal alternative with its high spatial (subnanometer scale) and high energy (below $0.2 \mathrm{eV}$ ) resolutions. ${ }^{20,21}$ Thanks to EELS, it has been recently possible to map LSPRs of different solid metal nanostructures, such as nanoparticles, ${ }^{22,23}$ nanorods/nanowires, ${ }^{24-26}$ nanoprisms, ${ }^{27}$ nanostars, ${ }^{28}$ nanodisks, $^{29}$ nanodecahedra, ${ }^{30}$ and nanocubes ${ }^{31-33}$ with high spatial resolutions. In addition to direct imaging of bright and dark plasmon modes in coupled nanostructures, ${ }^{29,34-36}$ EELS has also been used to study three-dimensional plasmonic properties $^{32}$ and quantum plasmonic effects (coupling effects) 


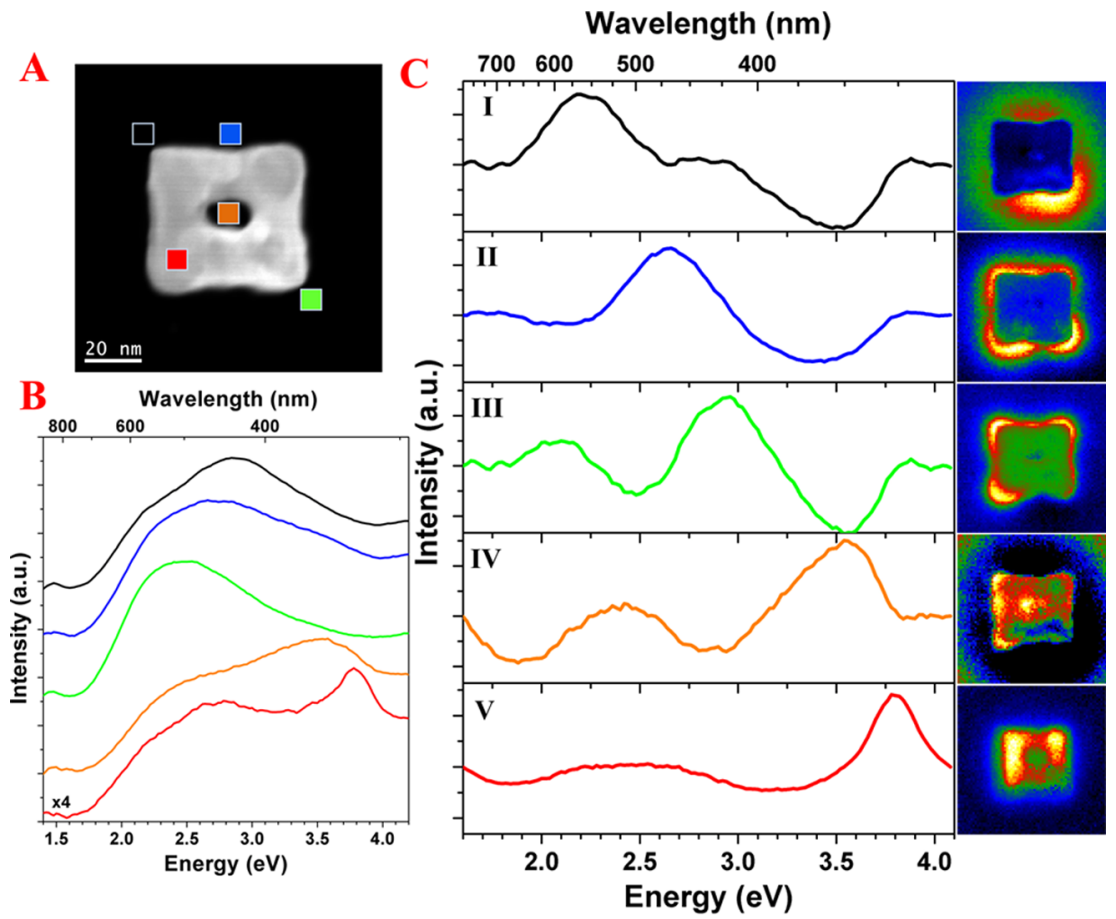

Figure 2. Plasmonic properties of the pinholed AuAg nanobox. (A) HAADF STEM micrograph of a $51 \mathrm{~nm}$ pinholed AuAg nanobox with a rectangular inner hole of $12 \mathrm{~nm} \times 17 \mathrm{~nm}$. (B) Selected area EEL spectra of the upper left and lower right corners, upper edge, center hole and bulk of the pinholed nanobox (areas are indicated in HAADF STEM micrograph with corresponding colors, which are 5 pixels by 5 pixels). (C) Spectra and abundance maps of five components obtained by VCA processing.

between nanoparticles. $^{23,37,38}$ Despite this huge effort, there is a clear lack of experimental studies dealing with the plasmonic properties of hollow metal nanostructures. To our knowledge, there is only one previous EELS study on polycrystalline nanoshells, ${ }^{39}$ but none on high quality, single crystalline hollow nanostructures such as commonly used nanoboxes and nanocages. Nevertheless, there are several theoretical studies dealing with the LSPR properties of individual and coupled AuAg hollow nanostructures by discrete dipole approximation (DDA) simulations, ${ }^{14,18,19,40}$ anticipating their enhanced properties; however, a detailed experimental characterization/ study at the nanoscale level along with a direct demonstration of their applicability is still missing in such advanced systems. ${ }^{41}$

Label-free optical sensing with plasmonic nanoparticles is based on the detection of adsorbate induced refractive index changes near or on the nanoparticles, which change the dielectric constant of the surrounding medium and can be measured by using UV-visible extinction spectroscopy., ${ }^{4,5,42}$ Despite the recent advances, there is still need for increased sensitivity to unleash the power of plasmonic sensing, before practical applications in the biological and medical testing devices can be released.

Here, we present a comprehensive EELS study on the evolution of LSPRs from solid Ag nanocubes to hollow AuAg nanoboxes/nanoframes. ${ }^{43}$ We have been able to finely tune plasmonic resonances generated due to these different structural features. We experimentally investigate the LSPRs of monocrystalline (i) Ag nanocubes, (ii) Ag@Au core-shell nanocubes, (iii) pinholed AuAg nanoboxes, (iv) single-walled AuAg nanoboxes, and (v) AuAg nanoframes, which are $\sim 50 \mathrm{~nm}$ in size, with sub-eV and nanometer resolutions by means of EELS spectrum imaging (SI). ${ }^{44}$ We have used a spectral unmixing (SU) based routine via vertex component analysis $(\mathrm{VCA})^{45-47}$ in order to process the EELS data sets, which is implemented in HyperSpy multidimensional data analysis toolbox. ${ }^{48} \mathrm{We}$ demonstrate, by direct measurements, the presence and distribution of different LSPR modes arising due to the interaction of inner and outer plasmon modes of the same hollow metal nanostructure and correlate our experimental findings with BEM simulations. ${ }^{49}$ We postulate that the experimentally obtained plasmon distributions are in agreement with the plasmon hybridization model, ${ }^{17}$ which represents the first experimental demonstrations at the nanoscale by EELS mapping in such hollow metal nanostructures. Finally, the enhanced plasmonic properties of hollow metal nanostructures are shown experimentally by measuring the sensitivity of singlewalled AuAg nanoboxes against conjugation events with bovine serum albumin (BSA) protein and its antibodies.

\section{RESULTS AND DISCUSSION}

Figure $1 \mathrm{~A}$ and $\mathrm{B}$ show representative sketches and high angle annular dark field (HAADF) STEM micrographs of the experimentally studied Ag nanocube and different AuAg nanostructures, respectively. The $\mathrm{AuAg}$ nanostructures are obtained by galvanic replacement reaction of $\mathrm{Ag}$ by $\mathrm{Au}^{3+}$, where the deposition of $\mathrm{Au}$ onto an $\mathrm{Ag}$ cube occurs as the $\mathrm{Ag}$ cube is emptied. Therefore, as the void size increases, the $\mathrm{Au} / \mathrm{Ag}$ ratio in the nanostructure also increases. In parallel, due to the high miscibility of $\mathrm{Au}$ and $\mathrm{Ag}$, they alloy to some degree spontaneously. ${ }^{43}$ See details on the AuAg nanostructures syntheses in Methods. Exploitation of plasmonic properties of hollow AuAg nanoboxes and nanocages has been the subject of intensive interest over the years as they have been used in many different application thanks to their enhanced plasmonic properties. $^{50,51}$ In parallel, efforts have been made to understand the optical properties of individual AuAg nanoboxes/ nanocages via single nanoparticle spectroscopy studies on 

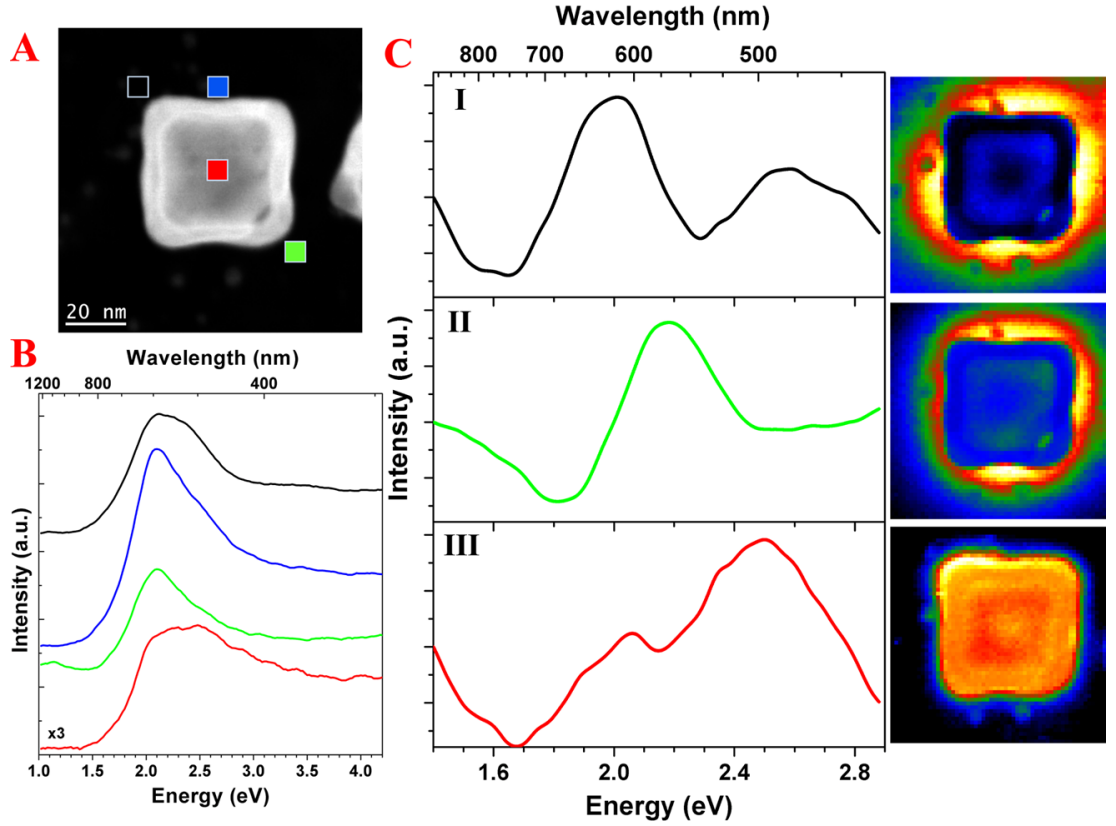

Figure 3. Plasmonic properties of the single-walled AuAg nanobox. (A) HAADF STEM micrograph of a $50 \mathrm{~nm}$ single-walled AuAg nanobox with 7 $\mathrm{nm}$ thick walls. (B) Selected area EEL spectra of the upper left and lower right corners, upper edge and center of the nanobox (areas are indicated in HAADF STEM micrograph, which are 5 pixels by 5 pixels). (C) Spectra and abundance maps of three components obtained by VCA processing.

various nanoboxes/nanocages. ${ }^{52,53}$ Here, we take these efforts to one step further by studying the spatially resolved plasmonic properties of individual hollow AuAg nanostructures at the nanoscale. Zero-loss peak (ZLP) subtracted low-loss EEL spectra, which are averaged over the EELS maps, of the nanostructures are presented in Figure $1 \mathrm{C}$, revealing the modulation of the averaged LSPRs induced by the morphological changes. As a general trend, LSPR peaks are shifted to lower energies with increasing void size. Bulk UV-vis spectra from each synthesis along with the low magnification micrographs are presented in Figure S1, confirming the shift of plasmon energies with increasing void size. Such an energy shift is the result of two main effects: (i) compositional effects due to AuAg alloying and (ii) morphological effects due to void formation. In order to better understand the effect of voids and to be able to distinguish them, if possible, from the compositional effects, we have simulated $50 \mathrm{~nm} \mathrm{Ag} \mathrm{nanoboxes}$ with various wall thicknesses (see Figure 1D), which suggests that the morphological changes are the dominant factor for the shift of plasmon resonance energies. As it can be seen in this figure, plasmon resonances shift to lower energies with increasing void size in accordance with the plasmon hybridization mechanism. The extent of the shift is higher for the thinner walls, that is, plasmon resonances barely shift for the 5 $\mathrm{nm}$ void but they shift about $0.8 \mathrm{eV}$ as the void size increases from 40 to $45 \mathrm{~nm}$ due to strong hybridization. It is also worth noting that their intensities, that is, loss probabilities, increase along with the increasing void size.

In the following, we describe the plasmonic properties of selected individual nanostructures. Detailed local EEL spectra of the $\mathrm{Ag}$ nanocube and $\mathrm{Ag} @ \mathrm{Au}$ core-shell nanocube and different components obtained by VCA processing from these nanostructures along with the relevant discussions about the shape and composition effects are presented in the Supporting Information.

Figure 2A shows the HAADF STEM micrograph of a $51 \mathrm{~nm}$ pinholed AuAg nanobox with a rectangular inner hole of $12 \mathrm{~nm}$ $\times 17 \mathrm{~nm}$. In Figure 2B, selected area EEL spectra obtained from different locations of the pinholed AuAg nanobox are shown, revealing the presence of wide peaks, which may contain several LSPR modes. The variances of these local EEL spectra (see, for instance, the spectra obtained from the upper left corner (in black) and lower right corner (in green)) suggest that the galvanic replacement reaction at this stage is taking place inhomogeneously. VCA processing revealed the distribution of five different components located at different energies, where, in some cases, more than one peak is observed for individual components caused by the inefficiency of the spectral unmixing routine which is thought to be due to the morphological and compositional complexity of the nanostructure. The components \#1, \#2, and \#3 can be associated with the LSPR modes related with the corner and/or edge excitations. The components \#4 and \#5 are resonances that can be related to the LSPR mode of $\mathrm{Ag}$ and bulk mode of $\mathrm{Ag}$, respectively. Above stated compositional variances are exhibited clearly with the abundance maps of these five different components (Figure $2 \mathrm{C})$. Figure $S 8$ shows the two additional components related to the background and interband transition contributions obtained during the processing of the pinholed AuAg nanoboxes.

Figure 3 shows the selected area EEL spectra obtained from different locations of the single-walled AuAg nanobox, which is $50 \mathrm{~nm}$ in size and has about $7 \mathrm{~nm}$ thick walls, suggesting that the plasmon peaks spanning between $\sim 2$ and $\sim 2.5 \mathrm{eV}$ have rather similar energies as one another, confirming the postulation that the hollow nanostructures would generate homogeneously distributed plasmon resonances. ${ }^{18,19}$ VCA processing shown in Figure 3C revealed the presence of three different components located at $\sim 2, \sim 2.2$, and $\sim 2.5 \mathrm{eV}$, where especially the abundance maps of the components \#1 and \#2 show their more or less homogeneous distribution with high intensities all around the nanobox. Plasmon distribution maps obtained by spectral filtering also suggest the homogeneous distribution of the plasmon resonances (see Figure S9). These maps revealing the spatial distribution of plasmon resonances in 


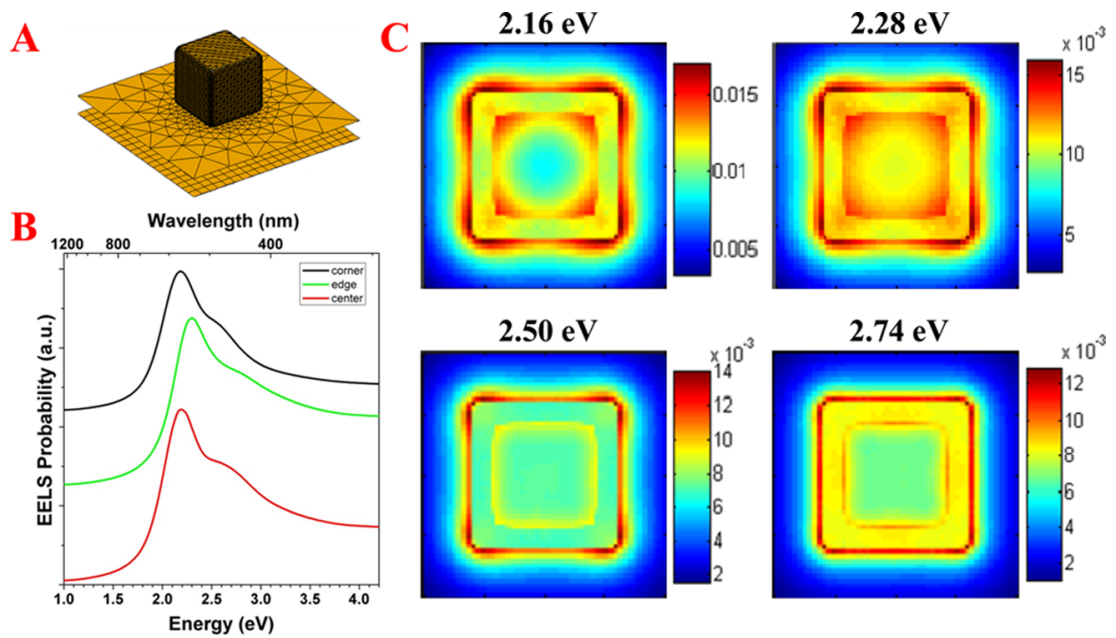

Figure 4. BEM simulations of the single-walled AuAg nanobox. (A) Structural model used during simulations. (B) Simulated local EEL spectra obtained from the corner, edge, and center of the nanobox. (C) Simulated plasmon maps of four different LSPR modes located at 2.16, 2.28, 2.50, and $2.74 \mathrm{eV}$.
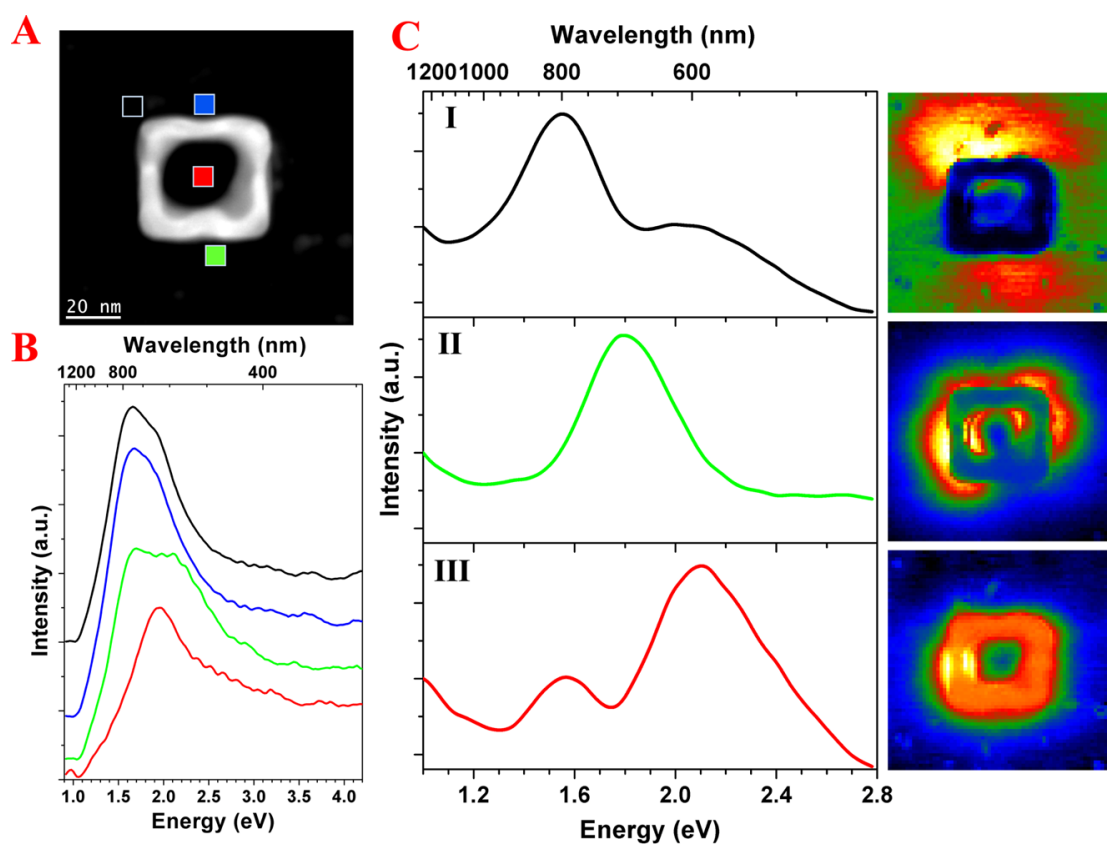

Figure 5. Plasmonic properties of the AuAg nanoframe. (A) HAADF STEM micrograph of a $48 \mathrm{~nm}$ AuAg nanoframe with $7 \mathrm{~nm}$ thick walls. (B) Selected area EEL spectra of the upper left corner, upper and lower edges, and center void of the nanoframe (areas are indicated in HAADF STEM micrograph, which are 5 pixel by 5 pixel). (C) Spectra and abundance maps of three components obtained by VCA processing.

such a hollow nanostructure may be the explanation of their enhanced plasmonic properties for different applications such as sensing $^{14,15}$ (vide infra), as all the surface of the nanobox acts like a continuous "hot-spot" with intense plasmon excitations.

In order to have a better understanding of the plasmonic properties of the AuAg nanobox, we have used BEM simulations. At least 10 different point analyses over the single-walled AuAg nanobox via STEM-energy dispersive X-ray spectroscopy (EDS) suggested that the AuAg nanoboxes are composed of $\sim 60 \% \mathrm{Au}$ and $\sim 40 \% \mathrm{Ag}$ with compositional variances of $\pm 7 \%$. We have assumed that the simulated nanobox, which is $50 \mathrm{~nm}$ in size with $7 \mathrm{~nm}$ thick walls, is composed of homogeneously distributed $60 \% \mathrm{Au}$ and $40 \% \mathrm{Ag}$. A second assumption was that the nanobox has no pores along the walls, as it is hardly possible to distinguish any pores along faces from a $2 \mathrm{D}$ projection. We have simulated a AuAg nanobox with sharp corners and have obtained qualitatively good agreement with the experimental plasmon resonance energies (see Figure S10). The LSPR mode located at $2.16 \mathrm{eV}$ is perfectly distributed all around the nanobox confirming the above-discussed plasmonic behavior of hollow nanostructures and presented experimental results. However, when we have a look at the LSPR mode located at $1.94 \mathrm{eV}$, we see that it is highly confined along the corners, which is not the case for the experimentally obtained abundance maps. In the light of these results, we have conducted BEM simulations on a AuAg nanobox with slightly rounded corners, as shown in Figure 4. As seen in the simulated local EEL spectra (Figure 4B) obtained from different locations of the structural AuAg nanobox model (Figure 4A), main plasmon resonances are located between 2.1 and $2.3 \mathrm{eV}$, which are slightly higher than the experimental values shown in Figure 3B. Such a small 
A

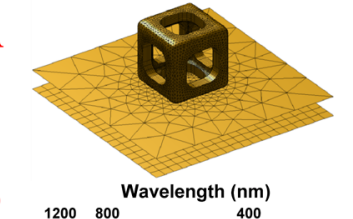

B

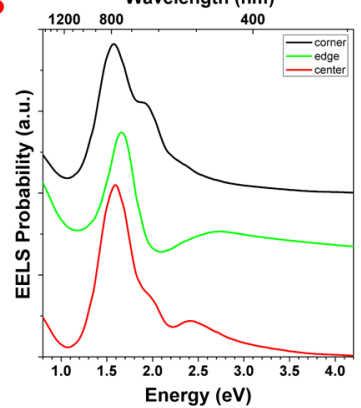

C
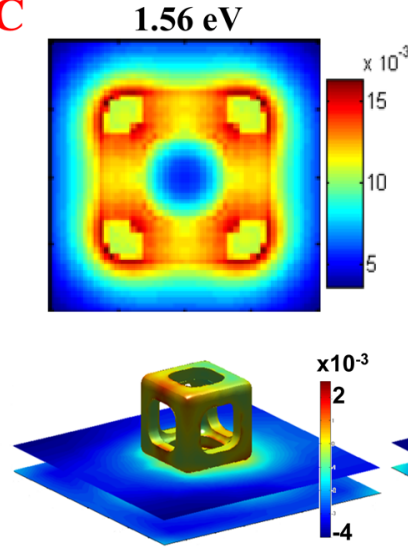

$1.68 \mathrm{eV}$

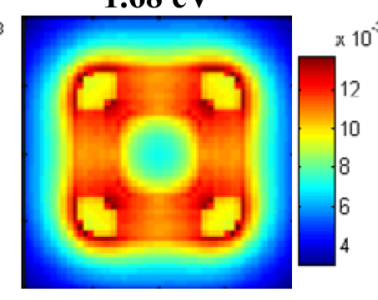

$1.94 \mathrm{eV}$

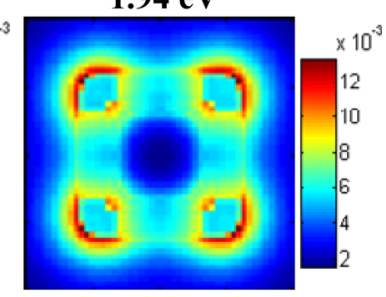

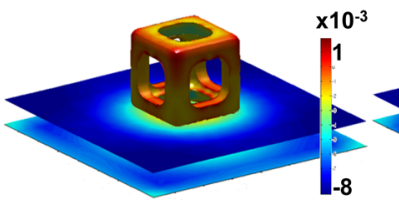

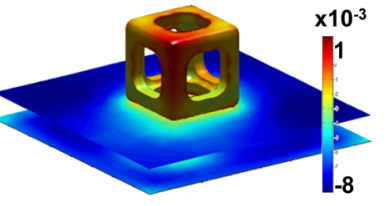

Figure 6. BEM simulations of the AuAg nanoframe. (A) Structural model used during simulations. (B) Simulated local EEL spectra obtained from the corner, edge, and center of the nanoframe. (C) Simulated plasmon maps of three different LSPR modes located at 1.56 , 1.68 , and 1.94 eV along with their corresponding induced electric field intensity distribution with an edge beam excitation in 3D (lower row).

difference can easily be related to the fact that an experimentally investigated nanobox has pores (see, for instance, the lower right corner in Figure 3A) which results in a shift to lower energies in accordance with the plasmon hybridization model ${ }^{17}$ (as clearly demonstrated in Figure 1D). The plasmon distribution maps shown in Figure 4C are well distributed around the nanobox and in good agreement with the experimentally observed ones. A homogeneous distribution of the plasmon resonances results in decreasing the number of plasmonic components as the difference between a corner mode and an edge mode is no longer applicable. The number of components for different nanostructures is in good agreement with this assumption, where the presence of seven components for the Ag nanocube (Figure S2) decreases to five for the pinholed AuAg nanobox (Figure 2), which further decreases to three for the single-walled AuAg nanobox (Figure 3) and AuAg nanoframe (Figure 5).

As the amount of $\mathrm{Au}$ further increased during the galvanic replacement reaction (see Methods for details), it is possible to obtain more complex nanostructures such as the nanoframe shown in Figure 5. The local EEL spectra obtained from different locations of the nanoframe are presented in Figure 5B, revealing that the main plasmon resonances are located between $\sim 1.7$ and $\sim 2 \mathrm{eV}$, which is lower than those observed for the AuAg nanobox. Such a shift is related to the increased $\mathrm{Au}$ content causing the creation of more voids in the AuAg nanoframes, ${ }^{53-55}$ resulting in a shift to lower energies in accordance with the plasmon hybridization mechanism. For instance, $\mathrm{Hu}$ et al. ${ }^{53}$ reported about $0.1 \mathrm{eV}$ red shift between the plasmon resonance energy of a AuAg nanobox and a AuAg nanocage via single nanoparticle spectroscopy, which was due to the presence of voids at the corners of the nanocage and therefore, a stronger hybridization. Moreover, the fact that the local EEL spectra obtained from the upper and lower edges are different from one another suggests that the voids along those faces, if there are any, are not identical. Figure 5C shows the spectra and abundance maps of the 3 components obtained by VCA processing. The component \#1, which is located at $\sim 1.5$ $\mathrm{eV}$, is mainly generated from the upper and lower parts of the nanoframe and the component located at $\sim 2.1 \mathrm{eV}$ is present at the nanoframe walls along with a highly intense interaction between the inner and outer part of the left edge. The component \#2 is located at $\sim 1.8 \mathrm{eV}$ and rather homogeneously distributed both inside and outside the nanoframe. It is worth noting that a local EEL spectrum obtained from the lower edge with higher energies than those of the other parts and the abundance map of the component \#2 with no excitation at the lower edge are concordant with each other, suggesting that this face should have smaller voids, than the other faces. These abundance maps showing the distribution of the plasmon resonances at the nanoscale clearly reveal the interaction of inner and outer plasmon fields in such hollow nanostructures. Figure S11 shows the plasmon distribution maps obtained by spectral filtering, which also reveal similar features with the abundance maps obtained by VCA, that is, confinement of high intensities at certain sides of the nanoframe for a given energy range, homogeneous distribution of main plasmon resonances, and interaction of the inner and outer plasmon resonances.

An ideal nanoframe would look like the one shown in Figure S12 with completely hollow faces, which generates plasmon resonances at low energies around $\sim 1 \mathrm{eV}$ (Figure S12B,C). However, the experimentally investigated nanoframe is significantly different than this ideal model. We have simulated a AuAg nanoframe, which is $48 \mathrm{~nm}$ in size with $7 \mathrm{~nm}$ thick walls and composed of homogeneous distribution of $70 \% \mathrm{Au}$ and $30 \% \mathrm{Ag}$, with $25 \mathrm{~nm}$ spherical pores along all faces (Figure 6A). Simulated local EEL spectra reveal that the main plasmon resonances are around $1.6 \mathrm{eV}$ with some shoulder peaks at higher energies. Distributions of three LSPR modes located at 1.56, 1.68, and $1.94 \mathrm{eV}$ are presented in Figure 6C along with their 3D distribution obtained by an edge beam excitation. As seen in these simulated plasmon maps, plasmon fields are rather homogeneously distributed around the nanoframe, especially the mode located at $1.68 \mathrm{eV}$. Simulated plasmon maps of three other modes located at higher energies of 2.24, 2.41, and 2.75 $\mathrm{eV}$, which could not be observed experimentally, are shown in Figure S13. Experimentally obtained and simulated plasmon resonance energies for the main three LSPR modes are qualitatively in good agreement with each other with error margins smaller than $0.2 \mathrm{eV}$. The differences between the distributions of the plasmon fields are related to the fact that experimentally investigated nanoframes do not have ideal symmetrical pores as in the simulated nanoframe. 

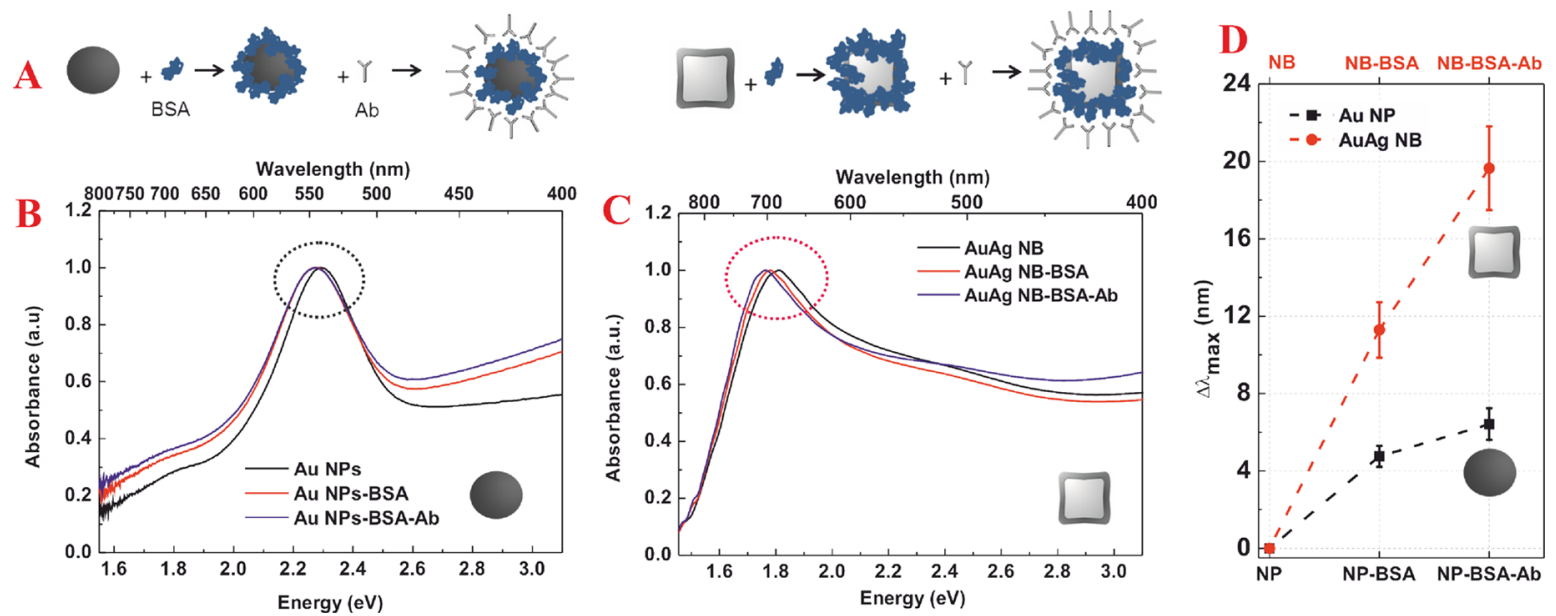

Figure 7. Biosensing with hollow nanostructures. (A) Schematic representation of the primary and secondary protein conjugation processes with spherical $\mathrm{Au}$ nanoparticles (left) and single-walled AuAg nanoboxes (right). UV-vis spectra of the unconjugated (black line) spherical Au nanoparticles (B) and single-walled AuAg nanoboxes (C) along with the spectra after conjugation with BSA (red line) and after the further addition of the $\mathrm{Ab}$ (blue line). Insets on $\mathrm{B}$ and $\mathrm{C}$ are plotted in $\mathrm{D}$, which is the redshift comparison between spherical Au nanoparticles and single-walled $\mathrm{AuAg}$ nanoboxes after the first $\mathrm{NP}-$ protein interaction and secondary protein- $\mathrm{Ab}$ interaction.

Up to this point, we have demonstrated the enhanced homogeneous distribution of the plasmon response in and around our nanoengineered hollow nanocuboids. In the following we will use these nanostructures in label-free sensing as a proof of concept for the enhanced plasmonic properties.

In this study, we detect the binding of antibodies to a protein corona ${ }^{56}$ formed on single-walled AuAg nanoboxes or on 50 $\mathrm{nm}$ spherical Au nanoparticles (Au NPs) as a benchmark ${ }^{57}$ for comparison. It is well-known that when the nanoparticles are dispersed in physiological media, they are immediately coated by proteins, forming a protein corona that may harden with time, ${ }^{58}$ becoming a hard protein shell that provides the biological identity ${ }^{59}$ of the nanoparticle, where native bovine serum albumin $(\mathrm{BSA})^{56}$ is one of the major compounds. Figure $7 \mathrm{~A}$ shows the schematic representation of the primary (with BSA) and secondary (with anti-BSA) protein conjugation processes with spherical solid nanoparticles and hollow nanoboxes, where the coverage of nanostructures with BSA and further attachment of antibodies are illustrated. The UVvisible spectra from the unconjugated Au NPs (Figure 7B) and single-walled AuAg nanoboxes (Figure 7C) along with their response to the BSA conjugation are shown in Figure 7. As can be seen in these UV-vis spectra, after incubation with BSA (shown in red), the position of the LSPR peak of spherical $\mathrm{Au}$ NPs does not change much (about $4 \mathrm{~nm}$ ), whereas the shift in the LSPR peak of the AuAg nanoboxes is clearly visible (about $12 \mathrm{~nm}$ ). Similar differences between the Au-BSA nanoparticles and single-walled AuAg-BSA nanoboxes are also observed during the second conjugation event when incubated with antibodies against BSA (shown in blue). Overall, it is clearly seen in Figure $7 \mathrm{D}$ that about $4 \times$ stronger shifts in the case of hollow AuAg nanoboxes are observed, especially in the second binding event, thanks to the enhancement of the localized electromagnetic field around the hollow nanoboxes that allows easy and direct detection of binding events in their vicinity. After experimentally obtaining an enhanced sensitivity with the hollow nanoboxes, we present the comparison of the sensitivities of different $\mathrm{Au}$ nanostructures on the basis of BEM simulations (Figure S14) and conclude that the voids along the faces are crucial for the enhanced plasmonic properties.

\section{CONCLUSIONS}

In summary, we have shown the localized surface plasmon resonance properties of several complex metal nanostructures: $\mathrm{Ag}$ nanocube, Ag@Au core-shell nanocube, pinholed AuAg nanobox, single-walled AuAg nanobox and AuAg nanoframe characterized by EELS technique in a monochromated STEM microscope with sub-eV energy resolutions at the nanoscale. We have used BEM simulations in order to better elucidate our experimental results. With the predetermined morphological and compositional assumptions, we have obtained good agreements between the experimentally collected EEL spectra and BEM simulated EEL spectra of the single-walled AuAg nanoboxes and AuAg nanoframes.

Hollow nanostructures have better plasmonic properties than their solid counterparts thanks to the interaction of the inner and outer plasmon fields, in compliance with the mechanism of plasmon hybridization. With the experimentally obtained plasmon maps of the present study, we intended to reveal first examples of spatially resolved interacting plasmon resonances in hollow AuAg nanoboxes and nanoframes at the nanoscale. Both experimental and computational studies have shown the homogeneous distribution of the plasmon resonances in these hollow nanostructures, which is suggested to be the key feature for the increased functionality. As a proofof-concept application for the enhanced plasmonic properties in the hollow nanostructures, we have conducted a sensing experiment where we have compared the response of commenly used spherical Au NPs and single-walled AuAg nanoboxes to sequential conjugation events with bovine serum albumin (BSA) and its antibodies (anti-BSA, Ab). In this way, we were able to observe that hollow nanoboxes show 4-fold increased sensitivity than spherical solid counterparts.

\section{METHODS}

Synthesis of AuAg Nanostructures. The hollow AuAg nanostructures used throughout the present work were synthesized via sequential galvanic replacement reaction and Kirkendall growth at room temperature, which is reported in ref 43. For the synthesis of AuAg nanostructures, $\mathrm{Ag}$ nanocubes were used as templates, which were synthesized by a modified 
polyol method. ${ }^{60,61}$ The straightforward morphological evolution from solid nanocubes to single-walled nanoboxes/nanoframes is due to the increasing extend of galvanic exchange between $\mathrm{Ag}$ and $\mathrm{Au}$. To be more precise, in a typical synthesis of single-walled $\mathrm{AuAg}$ nanoboxes $0.25 \mathrm{~mL}$ of $\mathrm{Ag}$ nanocubes $\left(\sim 10^{12} \mathrm{NPs} / \mathrm{mL}\right)$ were dispersed in $1 \mathrm{~mL}$ of milli-Q water, 1 $\mathrm{mL}$ of polyvinylpyrrolidone (PVP, $1 \mathrm{mM}$ by repeating unit), and $0.01 \mathrm{a} \mathrm{mL}$ of absorbic acid (AA, $0.1 \mathrm{mM}$ ) were added. Then, $0.3 \mathrm{~mL}$ of $\mathrm{HAuCl}_{4}(1 \mathrm{mM})$, was added through a syringe pump at a rate of $25 \mu \mathrm{L} / \mathrm{min}$ under stirring. Cetyltrimethylammonium bromide (CTAB, $1.0 \mathrm{~mL} 20 \mathrm{mM}$ ) was used as surfactant agent during the galvanic replacement reaction for the synthesis of Ag@Au core-shell nanocubes, AuAg pinholed nanoboxes and AuAg nanoframes. After the addition of the $\mathrm{HAuCl}_{4}$ solution the reaction was stirred for about $30 \mathrm{~min}$ at room temperature until the UV-vis spectra of the solution became stable. The sample was centrifuged at $8000 \mathrm{~g}$ for 10 min and the supernatant was discarded. The pellet was resuspended in $1 \mathrm{~mL}$ of milli- $\mathrm{Q}$ water for further characterization. As mentioned, this routine applies for the synthesis of intermediate nanostructures between the nanocubes and nanoboxes such as Ag@Au core-shell nanocubes and pinholed AuAg nanoboxes. The only difference there is the amount of $\mathrm{HAuCl}_{4}$ addition, which was $0.05 \mathrm{~mL}$ for the $\mathrm{Ag@Au} \mathrm{core-}$ shell nanocubes, $0.1 \mathrm{~mL}$ for the pinholed AuAg nanoboxes and $0.5 \mathrm{~mL}$ for AuAg nanoframes. In order to check the composition of the synthesized single-walled AuAg nanobox and AuAg nanoframe, we have conducted STEM-EDS analyses from at least 10 different points over the nanostructures using a FEI Tecnai (scanning) transmission electron microscope and presented the average $\mathrm{Ag}$ and $\mathrm{Au}$ values.

Solutions containing Ag nanocubes or AuAg nanostructures were ultrasonicated for about $20 \mathrm{~min}$ and deposited on $15 \mathrm{~nm}$ thick $\mathrm{Si}_{3} \mathrm{~N}_{4}$ membrane grids for STEM-EELS investigations. We have applied a hydrogen plasma cleaning by using a Plasma Etch plasma cleaner, at room temperature with a $\mathrm{H}_{2}$ flow of $1000 \mathrm{mT}$ and applied power of $100 \mathrm{~W}$, prior to EELS analyses in order to eliminate the presence of organic residues from the synthesis procedure.

EELS Acquisition and Data Processing. EELS analyses were performed using a probe-corrected FEI Titan 60-300 STEM equipment, operated at $80 \mathrm{kV}$ and equipped with a highbrightness X-FEG gun, a Wien filter monochromator and a Gatan Tridiem 866 ERS energy filter with a collection angle of $32 \mathrm{mrad}$ and a dispersion of $0.01 \mathrm{eV}$ per channel. Typical energy resolutions (full-width at half-maximum of the ZLP) of the measurements were better than $180 \mathrm{meV}$. EEL spectra were acquired using the spectrum imaging (SI) method ${ }^{44,62}$ in which a subnanometer electron probe was rastered over the area of interest with a constant displacement of 1-2 nm. After collecting the data, each EEL spectra were aligned along the Zero-Loss Peak (ZLP). In order to make surface plasmon peaks more visible for the presented local EEL spectra, a background subtraction (mainly for the ZLP and its tail) using the power law (PL) method in Gatan Digital Micrograph software was applied. $^{63}$

We have used a spectral unmixing (SU) based routine of vertex component analysis (VCA $)^{45-47}$ for spectral processing of the EELS data, which is developed by Nascimento and Bioucas-Dias. ${ }^{45}$ We have implemented the VCA code of ref 45 in HyperSpy ${ }^{48}$ multidimentional data analysis toolbox. Further details on the VCA routine and its application to EELS data can be found in the literature. ${ }^{45-47}$ During the spectral unmixing of each nanostructure, we have chosen the energy range on the basis of the local EEL spectra of the nanostructures considering the presence of plasmon peaks. It should be pointed out that we have used the EELS data sets without subtracting the ZLP during VCA processing. We have applied maximum number of components that generates meaningful data (i.e., not noise) and present them for each nanostructure. Note that background components are not presented except for the pinholed AuAg nanobox. For comparison purposes, we have processed the EELS data sets by using spectral filtering with Gaussian fitting and independent component analyses (ICA) by blind source separation (BSS) routines in HyperSpy (not shown here). ${ }^{64}$

Spectral filtering technique with energy windows, resembling the "EFTEM" technique, is also used to process EELS SI data sets. By using Gatan Digital Micrograph software, we have obtained the distribution of plasmon intensities, that is, plasmon loss probabilities, by applying an energy window of $0.2 \mathrm{eV}$ over the ZLP extracted EELS data of the single-walled $\mathrm{AuAg}$ nanobox and AuAg nanoframes. Obtained plasmon maps are normalized according to the maximum and minimum intensities among all the maps for each nanostructure.

Simulation. EELS simulations have been performed by using the boundary element method (BEM) which is developed by Garcia de Abajo and Howie. ${ }^{65,66}$ In particular, we have used a Matlab toolbox developed by Hohenester ${ }^{49}$ called MNPBEM, which calculates the plasmonic properties of metallic nanoparticles based on the BEM approach. The optical constants of the bulk metals have been taken from Johnson and Christy ${ }^{67}$ and modified according to ref 68 for AuAg alloys. We have also taken into account the size effects on the dielectric properties assuming an increase of the damping constant in the Drude model with reduction of the particle size due to the electron confinement effects. ${ }^{69}$ Throughout this paper, we have exploited the BEM simulations in order to elaborate the shape, composition and environmental (substrate) effects on the plasmonic properties of hollow AuAg nanostructures.

Label-Free Sensing. A $0.9 \mathrm{~mL}$ aliquot of nanoparticles $\left(10^{12} \mathrm{NPs} / \mathrm{mL}\right)$, spherical Au NPs or single-walled AuAg nanoboxes, dispersed in phosphate buffer $10 \mathrm{mM}$, were mixed with $0.1 \mathrm{~mL}$ of BSA $(1 \mathrm{mM})$ in phosphate buffer and placed in an incubator at $37{ }^{\circ} \mathrm{C}$ for $48 \mathrm{~h}$. After incubation of the nanoparticles, the UV-vis spectra were acquired. Finally, 0.02 $\mathrm{mL}$ of a $2 \mathrm{mg} / \mathrm{mL}$ solution of anti-BSA was added to the incubated nanoparticles and the UV-vis spectra were acquired. Each step was repeated three times for both spherical Au NPs and single-walled AuAg nanoboxes, and the shifts in the LSPR peaks are presented with an error margin. The reason that we used single-walled AuAg nanoboxes instead of nanoframes is that they can be synthesized with high abundances ( 90\%) compared to nanoframes $(\sim 50 \%){ }^{43}$

\section{ASSOCIATED CONTENT}

\section{Supporting Information}

The Supporting Information is available free of charge on the ACS Publications website at DOI: 10.1021/acsphotonics.5b00667.

UV-vis spectra of the nanostructures, detailed plasmonic properties of $\mathrm{Ag}$ nanocubes and $\mathrm{Ag}$ core-Au shell nanocubes, BEM simulations on the Ag nanostructures reporting the shape and substrate effects, BEM simulations of the AuAg nanobox and AuAg nanoframe with perfectly sharp corners, plasmon distribution maps 
of the AuAg nanobox and AuAg nanoframe obtained by spectral filtering and BEM simulated sensitivity of various $\mathrm{Au}$ nanostructures (PDF).

\section{AUTHOR INFORMATION}

\section{Corresponding Authors}

*E-mail: victor.puntes@icn.cat.

*E-mail: arbiol@icrea.cat.

\section{Author Contributions}

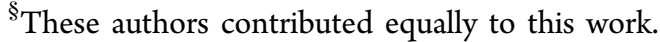

\section{Notes}

The authors declare no competing financial interest.

\section{ACKNOWLEDGMENTS}

J.A. and A.G. acknowledge the funding from Generalitat de Catalunya 2014 SGR 1638 and Spanish MICINN Project eATOM (MAT2014-51480-ERC). A.G. acknowledges the Turkish Ministry of National Education for the Ph.D. scholarship. J.P., N.G.B., and V.P. acknowledge financial support from the Generalitat de Catalunya 2014-SGR-612, Spanish MICINN (MAT2012-33330), and European Community (EU-FP7) through the FutureNanoNeeds project. N.G.B. thanks the Spanish MICINN for the financial support through the Juan de la Cierva program and European Commission for the Career Integration Grant (CIG)-Marie Curie Action. The microscopy works have been conducted in the "Laboratorio de Microscopias Avanzadas" at "Instituto de Nanociencia de Aragon - Universidad de Zaragoza” and Ernst Ruska-Centre for Microscopy and Spectroscopy with Electrons (ER-C). Authors thank the LMA-INA and ER-C for offering access to their instruments and expertise. Some of the research leading to these results has received funding from the European Union Seventh Framework Program under Grant Agreement 312483 - ESTEEM2 (Integrated Infrastructure Initiative - I3).

\section{REFERENCES}

(1) Barnes, W.; Dereux, A.; Ebbesen, T. Surface plasmon subwavelength optics. Nature 2003, 424, 824-830.

(2) Schuller, J.; Barnard, E. S.; Cai, W.; Jun, Y. C.; White, J. S.; Brongersma, M. L. Plasmonics for extreme light concentration and manipulation. Nat. Mater. 2010, 9, 193-204.

(3) Gramotnev, D. K.; Bozhevolnyi, S. I. Plasmonics beyond the diffraction limit. Nat. Photonics 2010, 4, 83-91.

(4) Mayer, K. M.; Hafner, J. H. Localized Surface Plasmon Resonance Sensors. Chem. Rev. 2011, 111, 3828-3857.

(5) Haes, A. J.; Haynes, C. L.; McFarland, A. D.; Schatz, G. C.; Van Duyne, R. P.; Zou, S. Plasmonic Materials for Surface-Enhanced Sensing and Spectroscopy. MRS Bull. 2005, 30, 368-375.

(6) Atwater, H. A.; Polman, A. Plasmonics for improved photovoltaic devices. Nat. Mater. 2010, 9, 205-213.

(7) Fang, N.; Lee, H.; Zhang, X. Sub-Diffraction-Limited Optical Imaging with a Silver Superlens. Science 2005, 308, 534-537.

(8) Sorger, V. J.; Zhang, X. Spotlight on plasmon lasers. Science 2011, 333, 709-710.

(9) Shalaev, V. M. Optical negative-index metamaterials. Nat. Photonics 2007, 1, 41-48.

(10) Chang, D. E.; Sorensen, A. S.; Hemmer, P. R.; Lukin, M. D. Quantum optics with surface plasmons. Phys. Rev. Lett. 2006, 97, 053002.

(11) Xia, Y.; Halas, N. J. Shape-controlled synthesis and surface plasmonic properties of metallic nanoparticles. MRS Bull. 2005, 30, $338-344$.
(12) Liz-Marzán, L. M. Tailoring Surface Plasmons through the Morphology and Assembly of Metal Nanoparticles. Langmuir 2006, $22,32-41$.

(13) Zhang, J.; Zhang, L. Nanostructures for surface plasmons. Adv. Opt. Photonics 2012, 4, 157-321.

(14) Mahmoud, M. A.; O’Neil, D.; El-Sayed, M. A. Hollow and Solid Nanoparticles in Sensing and in Nanocatalysis. Chem. Mater. 2014, 26, 44-58.

(15) Sun, Y.; Xia, Y. Increased sensitivity of surface plasmon resonance of gold nanoshells compared to that of gold solid colloids in response to environmental changes. Anal. Chem. 2002, 74, 52975305.

(16) Amendola, V.; Bakr, M. O.; Stellacci, F. A Study of the Surface Plasmon Resonance of Silver Nanoparticles by the Discrete Dipole Approximation Method: Effect of Shape, Size, Structure, and Assembly. Plasmonics 2010, 5, 85-97.

(17) Prodan, E.; Radloff, C.; Halas, N. J.; Nordlander, P. A Hybridization Model for the Plasmon Response of Complex Nanostructures. Science 2003, 302, 419-422.

(18) Mahmoud, M. A.; El-Sayed, M. A. Gold Nanoframes: Very High Surface Plasmon Fields and Excellent Near-Infrared Sensors. J. Am. Chem. Soc. 2010, 132, 12704-12710.

(19) Mahmoud, M. A.; Snyder, B.; El-Sayed, M. A. Surface Plasmon Fields and Coupling in the Hollow Gold Nanoparticles and SurfaceEnhanced Raman Spectroscopy. Theory and Experiment. J. Phys. Chem. C 2010, 114, 7436-7443.

(20) García de Abajo, F. J. Optical excitations in electron microscopy. Rev. Mod. Phys. 2010, 82, 209-275.

(21) Kociak, M.; Stephan, O. Mapping plasmons at the nanometer scale in an electron microscope. Chem. Soc. Rev. 2014, 43, 3865-3883.

(22) Bosman, M.; Keast, V. J.; Watanabe, M.; Maarof, A. I.; Cortie, M. B. Mapping surface plasmons at the nanometer scale with an electron beam. Nanotechnology 2007, 18, 165505.

(23) Scholl, J. A.; Koh, A. L.; Dionne, J. A. Quantum plasmon resonances of individual metallic nanoparticles. Nature 2012, 483, $421-427$.

(24) Nicoletti, O.; Wubs, M.; Mortensen, N. A.; Sigle, W.; van Aken, P. A.; Midgley, P. A. Surface plasmon modes of a single silver nanorod: an electron energy loss study. Opt. Express 2011, 19, 15371-15379.

(25) Guiton, B. S.; Iberi, V.; Li, S.; Leonard, D. N.; Parish, C. M.; Kotula, P. G.; Varela, M.; Schatz, G. C.; Pennycook, S. J.; Camden, J. P. Correlated Optical Measurements and Plasmon Mapping of Silver Nanorods. Nano Lett. 2011, 11, 3482-3488.

(26) Rossouw, D.; Couillard, M.; Vickery, J.; Kumacheva, E.; Botton, G. A. Multipolar Plasmonic Resonances in Silver Nanowire Antennas Imaged with a Subnanometer Electron Probe. Nano Lett. 2011, 11, 1499-1504.

(27) Nelayah, J.; Kociak, M.; Stephan, O.; Garcia de Abajo, F. J.; Tence, M.; Henrard, L.; Taverna, D.; Pastoriza-Santos, I.; Liz-Marzan, L. M.; Colliex, C. Mapping surface plasmons on a single metallic nanoparticle. Nat. Phys. 2007, 3, 348-353.

(28) Mazzucco, S.; Stephan, O.; Colliex, C.; Pastoriza-Santos, I.; LizMarzan, L. M.; Garcia de Abajo, F. J.; Kociak, M. Spatially resolved measurements of plasmonic eigenstates in complex-shaped, asymmetric nanoparticles: gold nanostars. Eur. Phys. J.: Appl. Phys. 2011, 54, 33512.

(29) Schmidt, F. P.; Ditlbacher, H.; Hohenester, U.; Hohenau, A.; Hofer, F.; Krenn, J. R. Dark Plasmonic Breathing Modes in Silver Nanodisks. Nano Lett. 2012, 12, 5780-5783.

(30) Myroshnychenko, V.; Nelayah, J.; Adamo, G.; Geuquet, N.; Rodriguez-Fernandez, J.; Pastoriza-Santos, I.; MacDonald, K. F.; Henrard, L.; Liz-Marzan, L. M.; Zheludev, N. I.; Kociak, M.; Garcia de Abajo, F. J. Plasmon Spectroscopy and Imaging of Individual Gold Nanodecahedra: A Combined Optical Microscopy, Cathodoluminescence, and Electron Energy-Loss Spectroscopy Study. Nano Lett. 2012, $12,4172-4180$.

(31) Mazzuco, S.; Geuquet, N.; Ye, J.; Stephan, O.; Van Roy, W.; Van Dorpe, P.; Henrard, L.; Kociak, M. Ultralocal Modification of Surface 
Plasmons Properties in Silver Nanocubes. Nano Lett. 2012, 12, 12881294.

(32) Nicoletti, O.; de la Peña, F.; Leary, R. K.; Holland, D. J.; Ducatti, C.; Midgley, P. A. Three-dimensional imaging of localized surface plasmon resonances of metal nanoparticles. Nature 2013, 502, 80-84.

(33) Goris, B.; Guzzinati, G.; Fernandez-Lopez, C.; Perez-Juste, J.; Liz-Marzan, L. M.; Trügler, A.; Hohenester, U.; Verbeeck, J.; Bals, S.; Van Tendeloo, G. Plasmon Mapping in $\mathrm{Au} @ \mathrm{Ag}$ Nanocube Assemblies. J. Phys. Chem. C 2014, 118, 15356-15362.

(34) Chu, M. W.; Myroshnychenko, V.; Chen, C. H.; Deng, J. P.; Mou, C. Y.; Garcia de Abajo, F. J. Probing Bright and Dark SurfacePlasmon Modes in Individual and Coupled Noble Metal Nanoparticles Using an Electron Beam. Nano Lett. 2009, 9, 399-404.

(35) Koh, A. L.; Fernandez-Dominguez, A. I.; McComb, D. W.; Maier, S. A.; Yang, J. K. W. High-Resolution Mapping of ElectronBeam-Excited Plasmon Modes in Lithographically Defined Gold Nanostructures. Nano Lett. 2011, 11, 1323-1330.

(36) Bigelow, N. W.; Vaschillo, A.; Iberi, V.; Camden, J. P.; Masiello, D. J. Characterization of the Electron- and Photon-Driven Plasmonic Excitations of Metal Nanorods. ACS Nano 2012, 6, 7497-7504.

(37) Scholl, J. A.; García-Etxarri, A.; Koh, A. L.; Dionne, J. A. Observation of Quantum Tunneling between Two Plasmonic Nanoparticles. Nano Lett. 2013, 13, 564-569.

(38) Tan, F. H.; Wu, L.; Yang, J. K. W.; Bai, P.; Bosman, M.; Nijhuis, C. A. Quantum Plasmon Resonances Controlled by Molecular Tunnel Junctions. Science 2014, 343, 1496-1499.

(39) Prieto, M.; Arenal, R; Henrard, L.; Gomez, L.; Sebastian, V.; Arruebo, M. Morphological Tunability of the Plasmonic Response: From Hollow Gold Nanoparticles to Gold Nanorings. J. Phys. Chem. C 2014, 118, 28804-28811.

(40) Qian, J.; Liu, C. X.; Wang, W. D.; Chen, J.; Li, Y. D.; Sun, Q. Effect of Edge Rounding on the Extinction Properties of Hollow Metal Nanoparticles. Plasmonics 2013, 8, 955-962.

(41) Parak, W. J. Complex Colloidal Assembly. Science 2011, 334, 1359-1360.

(42) Anker, J. N.; Hall, W. P.; Lyandres, O.; Shah, N. C.; Zhao, J.; Van Duyne, R. P. Biosensing with plasmonic nanosensors. Nat. Mater. 2008, 7, 442-453.

(43) González, E.; Arbiol, J.; Puntes, V. F. Carving at the Nanoscale: Sequential Galvanic Exchange and Kirkendall Growth at Room Temperature. Science 2011, 334, 1377-1380.

(44) Jeanguillaume, C.; Colliex, C. Spectrum-image: The next step in EELS digital acquisition and processing. Ultramicroscopy 1989, 28, 252-257.

(45) Nascimento, J. M. P.; Bioucas-Dias, J. Vertex component analysis: a fast algorithm to unmix hyperspectral data. IEEE T. Geosci. Remote 2005, 43, 898-910.

(46) Dobigeon, N.; Brun, N. Spectral mixture analysis of EELS spectrum-images. Ultramicroscopy 2012, 120, 25-34.

(47) Duchamp, M.; Lachmann, M.; Boothroyd, C. B.; Kovacs, A.; Haug, F.-J.; Ballif, C.; Dunin-Borkowski, R. E. Compositional study of defects in microcrystalline silicon solar cells using spectral decomposition in the scanning transmission electron microscope. Appl. Phys. Lett. 2013, 102, 133902.

(48) de la Peña, F.;Burdet, P.; Ostasevicius, T.; Sarahan, M.; Nord, M.; Fauske, V. T.; Taillon, J.; Eljarrat, A.; Mazzucco, S.; Donval, G.; Zagonel, L. F.; Walls, M.; Iyengar, I. hyperspy: HyperSpy 0.8.2; Zenodo, 2015; 10.5281/zenodo.28025.

(49) Hohenester, U. Simulating electron energy loss spectroscopy with the MNPBEM toolbox. Comput. Phys. Commun. 2014, 185, 1177-1187.

(50) Skrabalak, S. E.; Chen, J.; Au, L.; Lu, X.; Li, X.; Xia, Y. Gold nanocages for biomedical applications. Adv. Mater. 2007, 19, 31773184.

(51) Skrabalak, S. E.; Chen, J.; Sun, Y.; Lu, X.; Au, L.; Cobley, C. M.; Xia, Y. Gold Nanocages: Synthesis, Properties, and Applications. Acc. Chem. Res. 2008, 41, 1587-1595.

(52) Hu, M.; Petrova, H.; Sekkinen, A. R.; Chen, J.; McMellan, J. M.; Li, Z. Y.; Marquez, M.; Li, X.; Xia, Y.; Hartland, G. V. Optical
Properties of $\mathrm{Au}-\mathrm{Ag}$ Nanoboxes Studied by Single Nanoparticle Spectroscopy. J. Phys. Chem. B 2006, 110, 19923-19928.

(53) Hu, M.; Chen, J.; Marquez, M.; Xia, Y.; Hartland, G. V. Correlated Rayleigh Scattering Spectroscopy and Scanning Electron Microscopy Studies of Au-Ag Bimetallic Nanoboxes and Nanocages. J. Phys. Chem. C 2007, 111, 12558-12565.

(54) Zhang, J. Z. Biomedical applications of shape-controlled plasmonic nanostructures: A case study of hollow gold nanospheres for photothermal ablation therapy of cancer. J. Phys. Chem. Lett. 2010, $1,686-695$.

(55) Mahmoud, M. A.; El-Sayed, M. A. Metallic double shell hollow nanocages: The challenges of their synthetic techniques. Langmuir 2012, 28, 4051-4059.

(56) Casals, E.; Pfaller, T.; Duschl, A.; Janneke, O.; Puntes, V. Time Evolution of the Nanoparticle Protein Corona. ACS Nano 2010, 4, 3623-3632.

(57) Xia, F.; Zuo, X.; Yang, R.; Xiao, Y.; Kang, D.; Vallee-Belisle, A.; Gong, X.; Yuen, J. D.; Hsu, B. B. Y.; Heeger, A. J.; Plaxco, K. W. Colorimetric detection of DNA, small molecules, proteins, and ions using unmodified gold nanoparticles and conjugated polyelectrolytes. Proc. Natl. Acad. Sci. U. S. A. 2010, 107, 10837-10841.

(58) Casals, E.; Pfaller, T.; Duschl, A.; Oostingh, G. J.; Puntes, V. F. Hardening of the nanoparticle-protein corona in metal ( $\mathrm{Au}, \mathrm{Ag})$ and oxide $\left(\mathrm{Fe}_{3} \mathrm{O}_{4}, \mathrm{CoO}\right.$, and $\left.\mathrm{CeO}_{2}\right)$ nanoparticles. Small 2011, 7, 34793486.

(59) Monopoli, M. P.; Aberg, C.; Salvati, A.; Dawson, K. A. Biomolecular coronas provide the biological identity of nanosized materials. Nat. Nanotechnol. 2012, 7, 779-786.

(60) Skrabalak, S. E.; Au, L.; Li, X.; Xia, Y. Facile synthesis of Ag nanocubes and $\mathrm{Au}$ nanocages. Nat. Protoc. 2007, 2, 2182-2190.

(61) Zhang, Q.; Li, W.; Wen, L. P.; Chen, J.; Xia, Y. Facile synthesis of $\mathrm{Ag}$ nanocubes of 30 to $70 \mathrm{~nm}$ in edge length with $\mathrm{CF}_{3} \mathrm{COOAg}$ as a precursor. Chem. - Eur. J. 2010, 16, 10234-10239.

(62) Arenal, R.; de la Pena, F.; Stephan, O.; Walls, M.; Tence, M.; Loiseau, A.; Colliex, C. Extending the analysis of EELS spectrumimaging data, from elemental to bond mapping in complex nanostructures. Ultramicroscopy 2008, 109, 32-38.

(63) Egerton, R. F. Electron Energy-Loss Spectroscopy in the Electron Microscope; Plenum: New York, 1996.

(64) Genç, A. Plasmonic Nanoengineering in Hollow Metal Nanostructures: An Electron Energy-Loss Spectroscopy Study. Ph.D. Dissertation, Universitat Autònoma de Barcelona, 2015.

(65) García de Abajo, F. J.; Howie, A. Relativistic Electron Energy Loss and Electron-Induced Photon Emission in Inhomogeneous Dielectrics. Phys. Rev. Lett. 1998, 80, 5180-5183.

(66) García de Abajo, F. J.; Howie, A. Retarded field calculation of electron energy loss in inhomogeneous dielectrics. Phys. Rev. B: Condens. Matter Mater. Phys. 2002, 65, 1-17.

(67) Johnson, P. B.; Christy, R. W. Optical Constants of the Noble Metals. Phys. Rev. B 1972, 6, 4370-4379.

(68) Peña-Rodriguez, O.; Caro, M.; Rivera, A.; Olivares, J.; Perlado, J. M.; Caro, A. Optical properties of Au-Ag alloys: An ellipsometric study. Opt. Mater. Express 2014, 4, 403-410.

(69) Hövel, H.; Fritz, S.; Hilger, A.; Kreibig, U. Width of cluster plasmon resonances: Bulk dielectric functions and chemical interface damping. Phys. Rev. B: Condens. Matter Mater. Phys. 1993, 48, 1817818188. 